\title{
MONOCLONAL ANTIBODIES DEMONSTRATE THE ORGANIZATION OF AXONS IN THE LEECH ${ }^{1}$
}

\author{
SUSAN HOCKFIELD ${ }^{2}$ AND RONALD MCKAY \\ Cold Spring Harbor Laboratory, Cold Spring Harbor, New York 11724
}

Received June 21, 1982; Revised August 23, 1982; Accepted September 3, 1982

\begin{abstract}
Monoclonal antibodies have been generated that bind to subsets of neurons within the leech central nervous system (Zipser, B., and R. McKay (1981) Nature 289: 549-554). In this report we describe the binding patterns of monoclonal antibodies to subsets of axons in the leech using HRPimmunohistochemistry. Each antibody has a characteristic staining pattern in the connective, the large bundle of axons that runs the length of the nerve cord connecting each ganglion to its rostral and caudal neighbors. These staining patterns are consistent along the rostrocaudal axis of each animal, between animals of the same species, and, in many cases, between animals of different species. These results show that axonal position, like neuron cell body position, is a consistent feature of the organization of the leech central nervous system. Two antibodies bind to all of the axons in particular fascicles that are delimited by glial cell processes; another binds to single axons in fascicles that contain other, unstained axons. The grouping of antibody-identified axons into fascicles does not correlate in a simple way with the grouping of ncuron cell bodics identificd with the same antibody. The presence of one of these antigens on the surface of axons suggests a possible role in axon fasciculation. This report shows that molecular heterogeneity is a property of axons as well as of neuron cell bodies and demonstrates the organization of specific antibody-identified groups of axons within the connective.
\end{abstract}

The popularity of the leech as an experimental animal derives from the small number of stereotypically organized neuron cell bodies that constitute its central nervous system. With only minor variations, each of the 21 segmental ganglia is a replica of the others, containing approximately 400 neuron cell bodics, many of which have been identified physiologically, anatomically, and pharmacologically (see Muller et al., 1981). The relatively small number of neurons in a ganglion and their consistent positions, sizes, and shapes that can be resolved with the light microscope allow one to study the organization of the ganglia of the leech nervous system. In contrast, the connective, a bundle of axons that connects each $400-$ neuron ganglion to its neighbor, contains more than 5000 axons (Wilkinson and Coggeshall, 1975), many of whose profiles and positions can only be resolved at the electron microscopic level, making it far more difficult to study.

\footnotetext{
'We wish to thank E. Waldvogel for consistent and expert technical assistance. The cell lines used in this study were generated by $R$. M. and B. Zipser and were maintained by L. Kleina and C. Schley. This work was supported by National Institute of Neurological and Communicative Disorders and Stroke Grants 1RO1 NS18040 (S. H.) and 1RO1 NS17556 (R. M.) and by the Marie Robertson Fund.

${ }^{2}$ To whom correspondence should be addressed at Cold Spring Harbor Laboratory, P. O. Box 100, Cold Spring Harbor, NY 11724.
}

This study examines the organization of axons in the connective to determine if axonal positions are as consistently determined as neuron cell body position.

Recently, it has been shown that subsets of neurons within the leech nervous system can be identified with monoclonal antibodies (mABs) (Zipser and McKay, 1981), demonstrating that neurons are biochemically heterogeneous and can be identified by their molecular properties as well as by their anatomical and physiological properties. These observations suggest that mABs would allow a new approach to the study of neuronal organization. This study uses mABs to explore the anatomical distribution of specific, immunologically identified groups of axons in the connective. It shows that axonal position is a consistent feature of the leech nervous system and demonstrates molecules whose distribution correlates with some aspects of neuronal organization.

\section{Materials and Methods}

The monoclonal antibodies used in this study were raised by immunizing mice with homogenized adult leech nerve cords and have been described previously (with the exception of antibody Lan 4-2) (Zipser and McKay, 

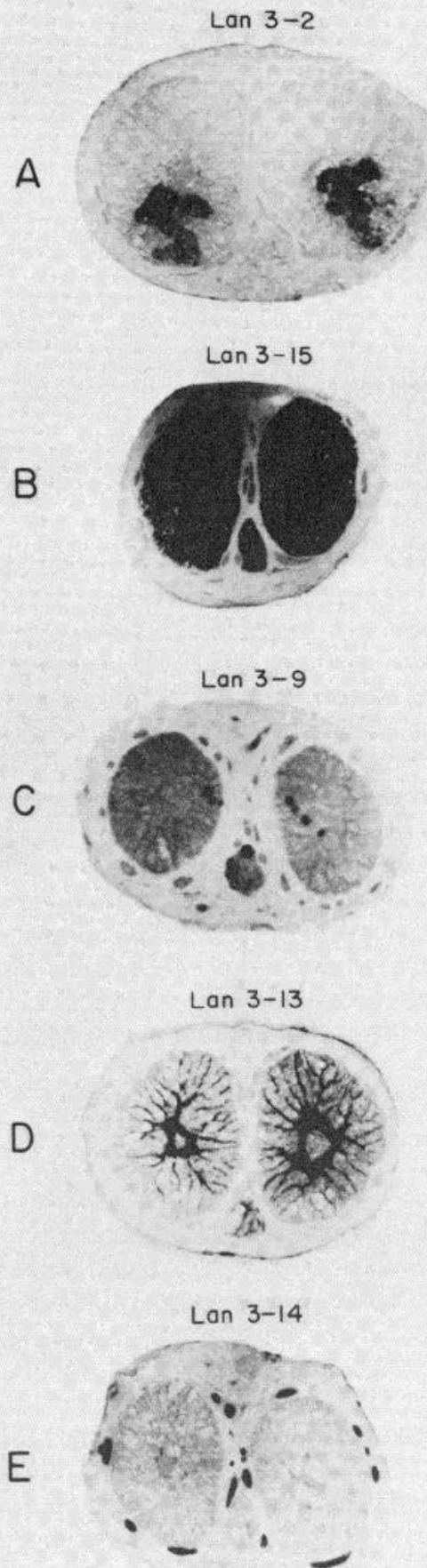

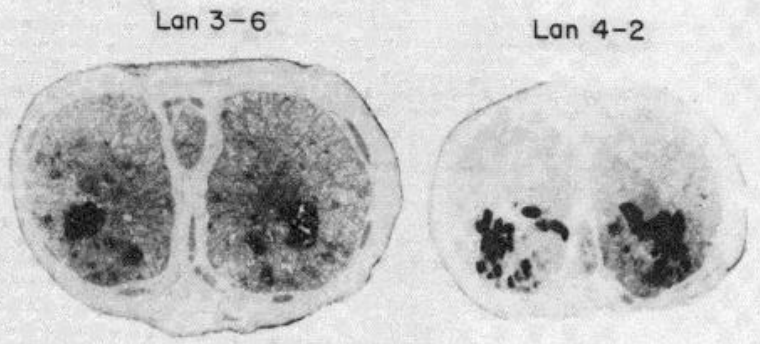

Lan $1-1$

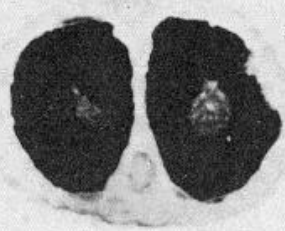

Lan 3-5

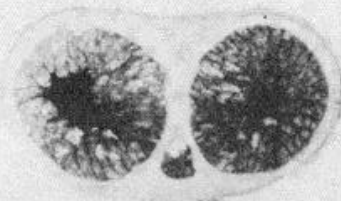

Figure 1. Two-micrometer-thick Epon/Araldite-embedded sections of connectives demonstrate the characteristic staining patterns of nine different monoclonal antibodies. In this and all following figures, dorsal is up in all transverse sections of the connective. A, Antibodies Lan 3-2, 3-6, and 4-2 stain some of the axons in the lateral part of each of the lateral bundles of axons but none in the medial bundle. $B$, Antibodies Lan 3-15 and 1-1 stain axons throughout each of the lateral bundles of axons. Lan 3-15 also stains axons in the medial bundle, while Lan 1-1 does not. C, Antibody Lan 3-9 stains axons in the medial bundle of axons and in the medial part of each of the lateral bundles. $D$, Antibodies Lan 3-13 and 3-5 stain the glial cell in the center of each of the lateral bundles of axons and glial cell processes in the lateral and medial bundles. Lan 3-5 stains the connective more extensively than does Lan 3-13. E. Antibody Lan 3-14 stains the muscle cell that runs in the connective tissue sheath and does not stain either axons or glia. Magnification $\times 950$.

1981). Antibodies were present in either supernatant from monoclonal cell lines (full strength) or from ascitic tumor fluid (at established dilutions) of mice injected with monoclonal cell lines.
Leeches (Haemopis marmorata or Hirudo medicinalis) were anesthetized in $10 \%$ alcohol and pinned in a dish, and a ventral incision was made through the skin and ventral blood sinus to expose the nerve cord. The 
A
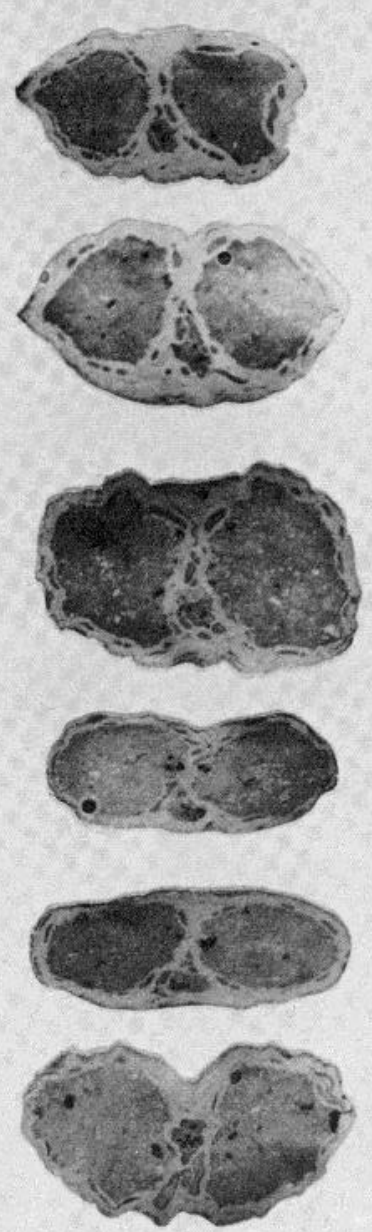

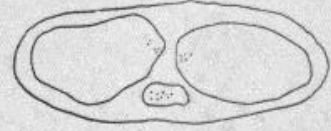

B
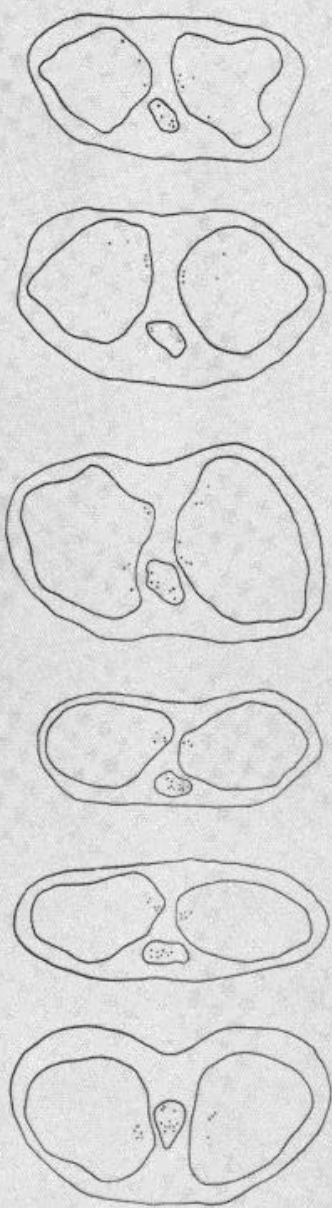

C
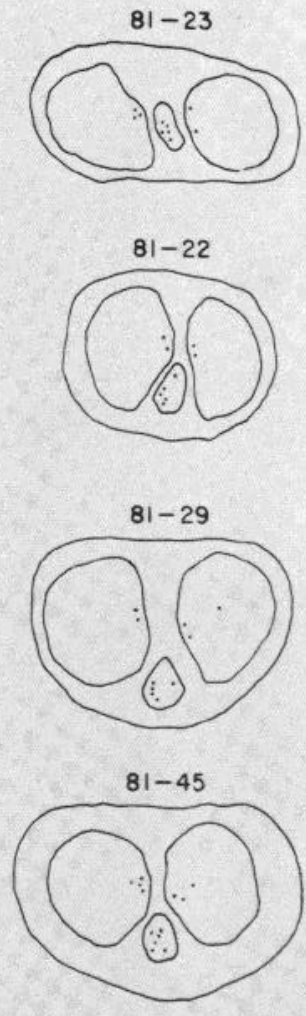

Figure 2. Connectives stained with Lan 3-9 illustrate the consistency in antibody-identified axonal position within an individual animal $(A$ and $B)$ and among animals of the same species $(C)$. A, Photomicrographs of Lan 3-9-stained 1- $\mu$ m-thick Epon/Aralditeembedded sections of the connective, each taken from a different segment of a single animal. $B$, Drawings of the photomicrographs in $A$ illustrate the positions of Lan 3-9-positive axons. The positions are consistent along the rostrocaudal axis of an animal. $C$, Drawings of connectives from four different animals demonstrate that the positions of Lan 3-9-stained axons are consistent among different animals.

nerve cord was flooded with fixative ( $4 \%$ paraformaldehyde in $0.1 \mathrm{M}$ phosphate buffer) for $30 \mathrm{~min}$ and then washed in buffer. All procedures were carried out at room temperature. Connectives from each segment were either dissected directly into buffer or embedded in a parallel array in $10 \%$ gelatin. Gelatin blocks containing the connectives were fixed in $4 \%$ paraformaldehyde and sectioned on a Vibratome at 50,100, or $150 \mu \mathrm{m}$. The sections were collected in phosphate buffer. Sections or whole connectives then were permeabilized by dehydration through graded alcohols into xylene and by rehydration back to buffer and were placed in $\mathrm{mAB}$ with $0.2 \%$ or $2 \%$ Triton X-100 overnight. Following a buffer wash, brief (15 min) fixation in 4\% paraformaldehyde, and another buffer wash, the sections were placed in a goat antimouse antibody conjugated to horseradish peroxidase (Cappel Labs) at a dilution of 1:30 with Triton X-100 for $2 \mathrm{hr}$ to overnight. Following a second series of buffer washes and fixation (20 min in 1\% glutaraldehyde), the horseradish peroxidase was visualized with $3,3^{\prime}$-diami- 


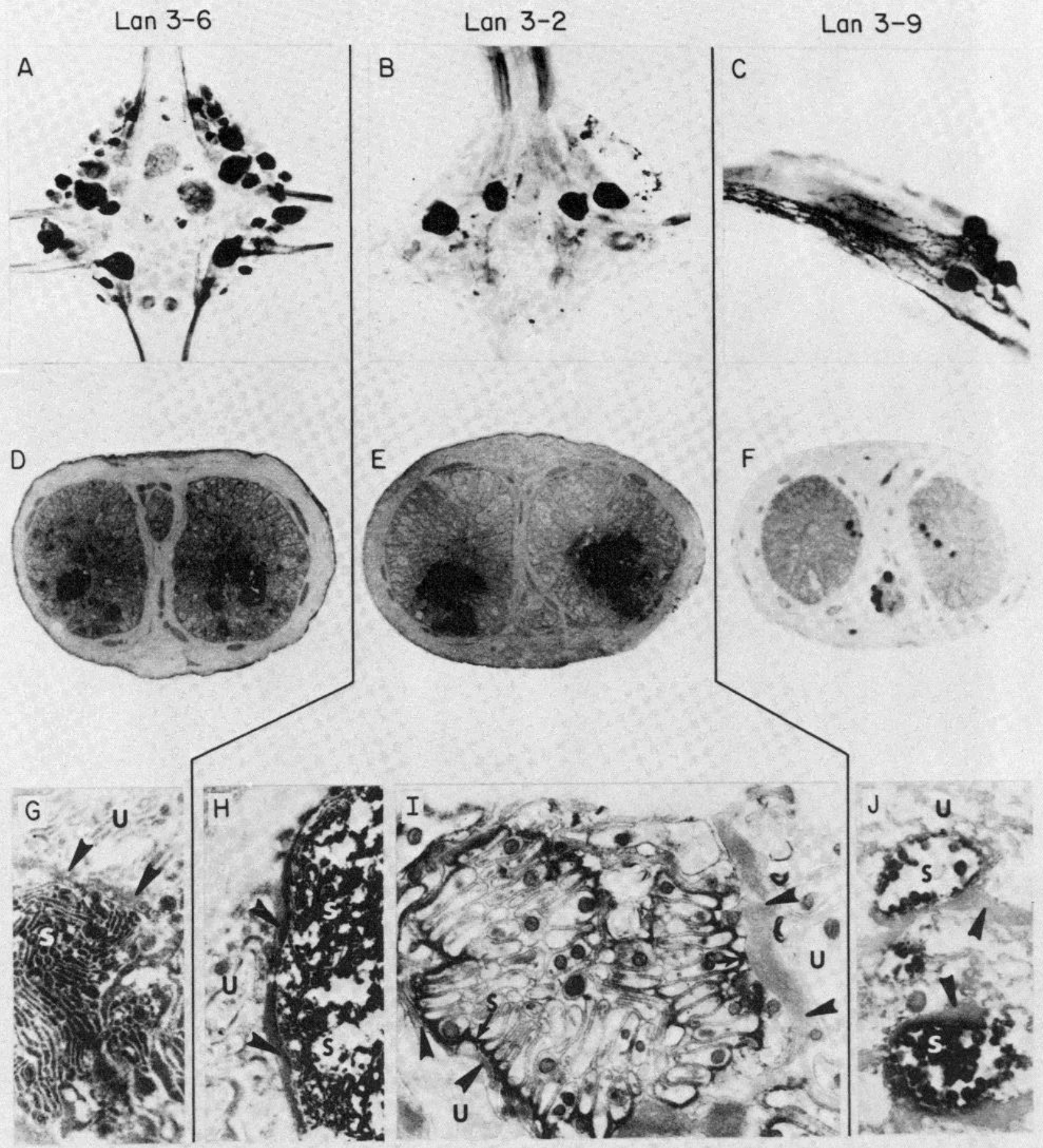

Figure 3. Comparisons between antibody-labeled cell bodies and axons show that neuronal cell body grouping does not entirely correlate with axonal grouping. Each column shows cell bodies and axons stained with one antibody: column 1, Lan 3-6; column 2, Lan 3-2; column 3, Lan 3-9. A, Standard segmental ganglia stained with Lan 3-6 contain approximately 40 stained cell bodies of various sizes and distributed throughout each ganglion (stained following procedures in Zipser and McKay, 1981). B, Standard segmental ganglia stained with Lan 3-2 contain four antibody-labeled cell bodies (nociceptive cells), two in each anterior compartment. $C$, Lan 3-9 stains five tightly grouped, $50-\mu \mathrm{m}$ cell bodies on each side of the supraesophageal ganglion. $D$, In $2-\mu$ mthick cross-sections of the connective, Lan 3-6-stained axons form fascicles in the lateral part of each lateral bundle of axons. $E$, Lan 3-2-stained axons form large fascicles in the ventrolateral part of each lateral connective. $F$, Lan 3-9-stained axons do not form fascicles and occupy small areas of the medial and lateral connectives. $G$, An electron micrograph of a xylene- and Triton X- 
nobenzidine (DAB) and $\mathrm{H}_{2} \mathrm{O}_{2}$ (Graham and Karnovsky, 1966). The sections were washed again in buffer and either mounted on slides and coverslipped or embedded in Epon/Araldite for the preparation of semithin sections.

For electron microscopy the above protocol was followed through the DAB step. In some cases $4 \%$ paraformaldehyde with $0.1 \%$ glutaraldehyde was used as the primary fixative and xylene and Triton X-100 were omitted. Following $\mathrm{DAB}$, the sections were washed, postfixed in $2 \% \mathrm{OsO}_{4}$, dehydrated, and embedded in Epon/Araldite. Thick sections ( 1 to $2 \mu \mathrm{m}$ ) were examined for antibody staining, sometimes counterstained with toluidine blue. Thin sections $(120 \mathrm{~nm})$ were examined without counterstain in a Philips EM2C electron microscope.

\section{Results}

The leech connective contains approximately 5000 axons (Wilkinson and Coggeshall, 1975) in three bundles: two large lateral bundles of axons and one small medial bundle. The medial bundle (Faivre's nerve) usually travels in the ventral portion of the connective, but its position is slightly variable; it can fuse with one of the lateral bundles or can occupy a dorsal position (Fernandez, 1978). A single glial cell is associated with each lateral bundle of axons. Its cell body lies in the center of each lateral bundle and the glial processes permeate the lateral (and medial) bundles, dividing the axons into fascicles (Coggeshall and Fawcett, 1964).

Each of the nine antibodies used in this study has a characteristic staining pattern in the connective of the leech, $H$. marmorata (Fig. 1). The five rows in Figure 1 show five different classes of antibody staining. Rows $A$ to $C$ show antibodies that stain axons; rows $D$ and $E$ show antibodies that stain glia or muscle. Row $A$ in Figure 1 illustrates three antibodies that stain ventral areas of the lateral bundles of axons and do not stain the medial bundle. Antibody Lan 3-2 stains a large area of the ventrolateral quadrant of each lateral bundle. Antibodies Lan 3-6 and 4-2 also stain axons in the ventrolateral quadrant, but the staining characteristics of each differ from those of Lan 3-2. Lan 3-6 stains a smaller area than does Lan 3-2, and Lan 4-2 staining is more discontinuous than that of Lan 3-2. Row $B$ in Figure 1 illustrates two antibodies (Lan 3-15 and 1-1) that bind to virtually all of the axons in each lateral bundle. Their staining patterns differ from one another in that Lan 3-15 stains the medial connective, while Lan 1-1 does not. In row $C$, antibody Lan 3-9 stains small areas in the medial bundle of axons and in the medial part of each of the lateral bundles. Row $D$ in Figure 1 illustrates two antibodies
(Lan 3-13 and 3-5) that bind to the central area of each lateral connective, conforming to the morphology of the connective glial cells. The glial cell body in the center of each lateral bundle appears to be stained as are the glial cell processes. Lan 3-5 appears to stain the connective more extensively than Lan 3-13, which might be due to Lan 3-5 staining both large and small glial processes, while Lan 3-13 may stain only the larger processes. In row $E$, antibody Lan 3-14 stains the muscle cell processes that run in the connective tissue capsule that surrounds the axon bundles. Lan 3-14 does not stain either axons or glial cells. Figure 1 illustrates that the overall staining pattern of each antibody is distinctive, enabling each to be unequivocally differentiated from the others. Even in the case where antibodies have similar staining patterns (i.e., those illustrated in Figure 1, row $A$ ), analysis of serial sections stained with different antibodies and repeated in a minimum of 10 separate experiments has confirmed their characteristic differences.

Identified neuron cell bodies occupy the same position in each standard segmental ganglion along the length of a leech and in segmental ganglia from all leeches of the same species (Nicholls and Baylor, 1968; Stuart, 1970). To determine if axonal position similarly is consistent in connectives from rostral to caudal segments, sections of the connective from different segments of a single leech were stained with one antibody (Fig. $2, A$ and $B$ ). The axons that stain with a given antibody have a similar distribution in the connective from all segments of a single animal. For example, in Figure 2, $A$ and $B$, antibody Lan 3-9 stains axons in the medial bundle of axons and in the medial part of each of the lateral bundles. The positions of stained axons are essentially consistent along the length of a leech.

To determine if these positions are consistent among leeches of the same species, comparisons were made among sections of connective from different leeches stained with the same antibody (Fig. $2 C$ ). Figure $2 C$ illustrates that staining patterns are the same among individual leeches of the same species. This consistency of axonal position along the rostrocaudal axis of a single animal and among animals of the same species has been found for all of the antibodies studied. In addition, in several cases, antibodies that cross-react with a second species of leech, $H$. medicinalis, stain axons in positions similar to those stained in $H$. marmorata (antibodies Lan 3-2, 4-2, 3-15, 1-1, and 3-9).

Some antibodies stain large areas of the connective, while others stain smaller areas (Fig. $3, D, E$, and $F$ ). At the light microscopic level it is not possible to resolve whether such areas constitute single axons or groups of

100-treated connective stained with Lan 3-5 shows that antibody-stained elements $(S)$ are separated from unstained elements $(U)$ by glial cell processes (arrowheads). Magnification $\times 15,000$. $H$, Lan 3-2-stained axons $(S)$ in electron micrographs of xyleneand Triton X-100-treated tissue also are separated from unstained axons $(U)$ by glial cell processes (arrowheads). Magnification $\times 15,000$. I, Lan 3-2 staining is associated with the surface of axons in tissue that has not been treated with xylene or Triton X-100. Here, a glial cell-delimited fascicle contains antibody-positive axons. Antibody staining $(S)$ is most prominent along the surface of axons opposed to glial cell processes (arrowheads). Magnification $\times 20,000 . J$, Lan 3-9-stained axons $(S)$ travel singly in the connective and do not form fascicles delimited from neighboring unstained axons ( $U$ ) by glial cell processes (arrowheads). Magnification $\times 15,000$. 
axons. At the electron microscopic level the large areas of the connective stained by antibodies Lan 3-6 and 3-2 are comprised of large numbers of stained axons (Fig. 3 , $G$ and $H$ ), while the smaller areas stained by Lan 3-9 are comprised of only a single or a few stained axons (Fig. $3 J)$. In many cases, the large bundles of axons stained by Lan 3-6 and 3-2 are delimited by glial cell processes (Fig. $3, G, H$, and $I$ ). When xylene and Triton X-100 are used to permeabilize the tissue during immunocytochemical procedures, all of the axons in such a bundle show antibody staining (Fig. 3, $G$ and $H$ ). When ultrastructure is preserved better by adding glutaraldehyde to the fixative and omitting Triton X-100 and xylene, the relationship between stained axons and glial cell processes is more apparent (Fig. 3I). Lan 3-2 stains the external membrane of many axons in a bundle; the axonal membranes directly opposed to glial cell processes are most consistently and intensely staining (Fig. $3 I$ ). More detailed ultrastructural information will be presented elsewhere (S. Hockfield et al., manuscript in preparation).

To determine if axon grouping correlates with cell body grouping, the staining patterns of three antibodies were examined in connectives and ganglia (Fig. 3). Lan 3-6 stains approximately 40 neuron cell bodies of differing sizes scattered throughout each standard segmental ganglion (Fig. $3 A$ ) and stains bundles of axons in the mediolateral part of each lateral connective (Fig. $3 D$ ). Lan 32 stains 4 neuron cell bodies in each standard segmental ganglion (Fig. $3 B$ ) and bundles of axons in the ventrolateral part of each lateral connective (Fig. $3 E$ ). In both of these cases the bundles of stained axons are often delimited by glial cell processes (Fig. $3, G$ and $H$ ). In contrast to these two examples, Lan 3-9 only stains 10 neuron cell bodies bilaterally arranged in two groups of 5 neurons in the supraesophageal ganglion (Fig. $3 \mathrm{C}$ ). These cell bodies are all the same size $(50 \mu \mathrm{m})$ and are closely grouped together. The axons stained with Lan 3-9 (Fig. 3, $F$ and J) travel singly through the connective and are not isolated as a group from neighboring axons by glial cll processes (Fig. 3J).

\section{Discussion}

In this study we have examined the organization of axons in the connective of the leech using monoclonal antibodies. This approach has allowed us to examine the organization of particular subsets of axons along the entire rostrocaudal extent of a single animal and among different individual animals. The power of the technique lies in its ability to identify a group of neural elements that share a specific antigen, and this greatly facilitates the task of anatomically identifying a particular class of cell bodies or processes for study.

We have shown here that axonal position is essentially consistent in the connective from different segments along the rostrocaudal axis of the leech and among leeches of the same species $(H$. marmorata). Some antibodies cross-react with leeches of another species $(H$. medicinalis) and in most of these cases the axonal positions are the same as in $H$. marmorata, the species against which the antibodics were raised. A number of reports have demonstrated that the segmental ganglia are similarly organized in a single leech, between leeches of the same species (Nicholls and Baylor, 1968; Stuart,
1970), and, in many cases, between leeches of different species (Keyser and Lent, 1977; Macagno, 1980). This study shows that location stereotypy is not solely a property of neuron cell bodies but also of neuron cell processes.

This study also shows that areas of the connective are occupied by fascicles of axons carrying a particular antigen. Two such antibodies have been described in detail here. These observations show that the axons within some fascicles have a common molecular marker. This may be a general feature of axonal organization in the connective, in which case there might be a molecular marker for each fascicle of the connective. Interestingly, antibody Lan 3-9 stains single axons in the connective (Fig. 3, $F$ and $J$ ) and each of these single axons often lies within a fascicle that contains other unlabeled axons. If all of the axons within a particular fascicle share a molecular marker, then each of the Lan 3-9-stained axons might also contain a molecule common to all axons in the fascicle it occupies.

An antibody that recognizes bundles of axons does not necessarily recognize clustered groups of cell bodies and vice versa (Fig. 3). Antibody Lan 3-9 recognizes neuron cell bodies that are anatomically homogeneous and tightly grouped and recognizes axons that are dispersed in the connective. In contrast, Lan 3-6 recognizes 40 cell bodies that are anatomically heterogeneous and are dispersed in the ganglia and axons that form fascicles in the connective. While both cell bodies in the ganglia and axons in the connective are stereotypically organized, there is no simple relationship between the organization of these two structures. This finding indicates that the factors that determine axon and cell body position either are not the same or operate in different ways in different locations. During development, cell body position in the ganglion is achieved before axon position in the conneclive (Kuwada and Kristan, 1981). The factors that govern cell body position, then, may be independent of those governing axon position. The presence of a particular molecule (perhaps associated with the axolemma as demonstrated here for Lan 3-2, Fig. 3I) may guide an axon to its position with a fascicle. It is of interest here that antibody-labeled fascicles of axons are often delimited by glial cell processes. The recognition and guidance might be mediated by interactions between axonal and glial cell surfaces.

We have shown here that molecular heterogeneity is a property of axons as well as of neuron cell bodies. Monoclonal antibodies have been used to examine the organization of axons and have shown that axonal position is a consistent feature of the leech nervous system. The grouping of axons into fascisles is a feature of both invertebrate and vertebrate nervous systems. Developing neurons in the grasshopper grow out along determined routes to form axon fascicles (Bate, 1976; Goodman et al., 1982; Keshishian and Bentley, 1981). In the adult vertebrate nervous system, axons with related functions often form fascicles, such as the fasciculi gracilis and cuneatus and the corticospinal tract of the spinal cord (Coggeshall and Willis, 1978). In the present study we have shown that axon fascicles can carry specific molecular markers. These findings suggest that such markers may be a feature of all nervous systems; that 
the axons that comprise a fascicle may do so because they share a particular molecular characteristic. While the precise biochemical factors that might encode this positional information are not known, the monoclonal antibody technology gives us a means to identify molecularly homogeneous subsets of axons and to search further for biochemical factors which have properties we expect of molecules that organize axons into specific, stereotyped arrays.

\section{References}

Bate, C. M. (1976) Pioneer neurons in an insect embryo. Nature 260: 54-56.

Coggeshall, R. E., and D. W. Fawcett (1964) 'The fine structure of the CNS of the leech, Hirudo medicinalis. J. Neurophysiol. 27: 229-28.

Coggeshall, R. E., and W. D. Willis (1978) Sensory Mechanisms of the Spinal Cord, Plenum Press, New York.

Fernandez, J. (1978) Structure of the leech nerve cord: Distribution of neurons and organization of fiber pathways. J. Comp. Neurol. 180: 165-192.

Goodman, C. S., J. A. Raper, R. K. Ho, and S. Chang (1982) Pathfinding by neuronal growth cones in grasshopper embryos. Symp. Soc. Dev. Biol. 40: 275-360.

Graham, R. C., and M. J. Karnovsky (1966) The early stages of absorption of injected horseradish peroxidase in the proximal tubules of the mouse kidney: Ultrastructural cytochemistry by a new technique. J. Histochem. Cytochem. 14: 291-302.

Keshishian, H., and D. Bentley (1981) Multiple pioneer neurons establish (in vitro and in embryo culture) major nerve trunks followed preferentially by identified neurons. Soc. Neurosci. Abstr. 7: 347.

Keyser, K. T., and C. M. Lent (1977) On neuronal homologies within the CNS of leeches. Comp. Biochem. Physiol. (A) 58: $285-297$.

Kuwada, J. Y,, and W. B. Kristan (1981) Development of identified neurons in the leech. Soc. Neurosci. Abstr. 7: 3.

Macagno, E. R. (1980) Number and distribution of neurons in leech segmental ganglia. J. Comp. Neurol. 190: 283-302.

Muller, K. J., J. G. Nicholls, and G. S. Stent, eds. (1981) Neurobiology of the Leech, Cold Spring Harbor Laboratory, Cold Spring Harbor, New York.

Nicholls, J. G., and D. A. Baylor (1968) Specific modalities and receptive fields of sensory neurons in CNS of the leech. J. Neurophysiol. 31: 740-756.

Stuart, A. E. (1970) Physiological and morphological properties of motor neurons in the central nervous system of the leech. J. Physiol. (Tond.) 209: 627-646.

Wilkinson, J. M., and R. E. Coggeshall (1975) Axonal numbers and sizes in the connectives and peripheral nerves of the leech. J. Comp. Neurol. 162: 387-396.

Zipser, B., and R. McKay (1981) Monoclonal antibody distinguishes individual leech neurons. Nature 289: 549-554. 